\title{
Cortisol, resposta glicêmica e secreção de insulina em equinos clinicamente sadios submetidos a sessões de hipóxia normobárica
}

\author{
Serum cortisol, glycemic response and insulin secretion in healthy horses exposed to normobaric \\ hypoxia sessions
}

\author{
Diego De Gasperi ${ }^{\mathrm{I}}$ Eduardo Almeida da Silveira ${ }^{\mathrm{I}}$ Diego Rafael Palma da Silva ${ }^{\mathrm{I}}$ \\ Roberta Carneiro da Fontoura Pereira ${ }^{\mathrm{I}}$ Luiz Osório Cruz Portela ${ }^{\mathrm{I}}$ \\ Karin Erica Brass ${ }^{\mathrm{III}}$ Flávio Desessards De La Corte ${ }^{\mathrm{II}}{ }^{*}$
}

RESUMO

\begin{abstract}
Os níveis séricos de cortisol, resposta glicêmica e secreção de insulina foram avaliados em equinos não exercitados submetidos a sessões de hipóxia normobárica. Cavalos adultos sadios $(n=8)$ foram selecionados após exame físico e submetidos ao teste de tolerância à glicose intravenosa $\left(0,5 \mathrm{~g} \mathrm{~kg}^{-1}\right)$ (TTGIV). Amostras de sangue $(n=11)$ foram coletadas entre 0 e 180 minutos após a administração de glicose para avaliar a resposta glicêmica e a secreção de insulina. Os animais foram então expostos a sessões de uma hora de hipóxia duas vezes ao dia, totalizando 43 sessões, com concentração decrescente de $\mathrm{O}_{2}$ de 17 a $12 \%$. A frequência cardíaca e as amostras sanguíneas para a determinação do cortisol sérico foram coletadas antes e a cada 15 minutos durante as sessões $1,4,6,8,10,14,22$ e 43. A TTGIV foi repetida ao final do estudo. Os níveis de cortisol sérico foram maiores na sessão 1 ( $\mathrm{S} 1)\left(17 \% \mathrm{O}_{2}\right)$ quando comparados aos níveis da $S 10\left(13 \% \mathrm{O}_{2}\right)$ e da $S 43\left(12 \% \mathrm{O}_{2}\right)(P<0,05)$. A curva glicêmica de equinos clinicamente sadios não submetidos a exercício não é alterada pela exposição a 43 sessões de hipóxia normobárica. $O$ aumento na secreção de insulina, acompanhada de diminuição da frequência cardíaca e cortisol sérico sugerem uma adaptação dos cavalos ao modelo de hipóxia utilizado.
\end{abstract}

Palavras-chave: cavalos, hipóxia intermitente, glicemia, estresse, altitude.

\section{ABSTRACT}

The cortisol levels, glycemic response and insulin secretion were evaluated in non-exercised horses submitted to sessions of normobaric hypoxia. Healthy adult horses $(n=8)$ were selected after physical examination and underwent an intravenous glucose tolerance test $\left(0.5 \mathrm{~g} \mathrm{~kg}^{-1}\right)$ (IVGTT). Blood samples $(n=11)$ were collected between 0 and 180 minutes after glucose administration to evaluate glycemic response and insulin secretion. Then, they were exposed to 1-hour hypoxia sessions twice a day, reaching a total of 43 hypoxia sessions, with $\left[\mathrm{O}_{2}\right]$ decreasing from 17 to $12 \%$. Heart rate and serum samples to measure cortisol were collected before and every 15 minutes during the sessions 1, 4, 6, 8, 10, 14, 22 and 43. The IVGTT was repeated at the end of the study. The serum cortisol levels were higher in session $1(\mathrm{~S} 1)\left(17 \% \mathrm{O}_{2}\right)$ when compared to the levels of $\mathrm{S} 10\left(13 \% \mathrm{O}_{2}\right)$ and $S 43\left(12 \% \mathrm{O}_{2}\right)(P<0.05)$. The glycemic curve in resting healthy horses is not altered by exposure to 43 normobaric hypoxia sessions. The increase in insulin secretion, followed by decreased heart rate and serum cortisol suggest an adaptation of the horses to the hypoxia model used.

Key words: horses, intermittent hypoxia, glycemia, stress, altitude.

\section{INTRODUÇÃO}

A exposição a um ambiente de hipóxia aguda ou crônica pode influenciar no metabolismo da glicose e hormônios relacionados. Entretanto, os resultados publicados até o momento ainda são inconsistentes. Isso provavelmente ocorre pela diversidade entre espécies, grau e duração da hipóxia ou como consequência da complexidade dos mecanismos regulatórios do metabolismo (CHEN et al., 2007).

'Programa de Pós-graduação em Medicina Veterinária, Universidade Federal de Santa Maria (UFSM), Santa Maria, RS, Brasil. "Laboratório de Fisiologia e Performance Humana, UFSM, Santa Maria, RS, Brasil.

"IIDepartamento de Clínica de Grandes Animais, Hospital Veterinário, UFSM, 97105-900, Santa Maria, RS, Brasil. e-mail: delacorte2005@yahoo.com.br*Autor para correspondência 
Estudos em diversas espécies demonstraram que a exposição a diferentes condições de hipóxia pode causar aumento da resistência à insulina (CHENG et al., 1997; BRAUN et al., 2001; POLOTSKY et al., 2003; IIYORI et al., 2007). Um dos hormônios capazes de causar tal efeito é o cortisol (ANDREWS \& WALKER, 1999), que pode estar com seus níveis circulantes aumentados quando da exposição aguda ou crônica de indivíduos a baixas concentrações de oxigênio $\left(\left[\mathrm{O}_{2}\right]\right)$ (HUMPELER et al., 1980; COSTE et al., 2005).

A elevação dos níveis de cortisol no sangue é um bom indicador que pode ser usado para avaliar se um equino sofre de estresse (ALEXANDER \& IRVINE, 1998). As glândulas adrenais possuem o papel principal nas reações hormonais envolvidas nessa condição, por estarem diretamente ligadas ao eixo hipotálamohipófise-adrenal (HHA) e ao sistema simpático. Apesar de muitos hormônios estarem envolvidos nessa resposta, o primeiro mecanismo endócrino de defesa é o aumento da secreção de glicocorticóides e catecolaminas (MÖSTL \& PALME, 2002). A liberação desses é consequência do aumento de ACTH circulante, que possui mecanismos multifatoriais de regulação com a liberação de fatores estimulantes do hipotálamo, como o hormônio liberador de corticotrofina (CRH) e a vasopressina (VP), e a influência inibitória dos glicocorticóides quando em estresse crônico (AGUILERA, 1994).

No passado, a maioria das pesquisas apontou para os efeitos da hipóxia aguda ou crônica no intuito de estudar a adaptação em resposta às altas altitudes. Mais recentemente, alguns estudos têm buscado desvendar os efeitos da exposição à hipóxia intermitente $(\mathrm{HI})$, objetivando explorar o valor terapêutico em pacientes e a influência potencial no desempenho de atletas (NEUBAUER, 2001).

Na medicina, a HI já foi aplicada em pacientes com doença pulmonar obstrutiva crônica (DPOC) (BURTSCHER et al., 2009), hipertensão, processos inflamatórios, doença de Parkinson, entre outras (SEREBROVSKAYA, 2002). Em animais, a HI mostrou ter efeito antiarrítmico em casos de isquemia aguda do miocárdio em ratos (MEERSON et al., 1987) e para proteger o miocárdio de cães com infarto experimentalmente induzido (ZONG et al., 2004).

Em equinos, as principais causas em ordem decrescente de diminuição de desempenho atlético são as doenças musculoesqueléticas, respiratórias e cardiovasculares. Dentre as quais, muitas são semelhantes às afecções citadas anteriormente, como DPOC, arritmias cardíacas e doenças do miocárdio (HINES, 2004). Além dessas, doenças como a miopatia por acúmulo de polissacarídeos, na qual os cavalos apresentam um aumento na captação da glicose pelas células musculares e um aumento da sensibilidade à insulina (DE LA CORTE et al., 1999) podem ser alvo de futuros estudos para o uso potencial da $\mathrm{HI}$ em medicina veterinária.

O objetivo do presente estudo foi avaliar o efeito da hipóxia normobárica intermitente na resposta glicêmica, secreção de insulina e nos níveis de cortisol sanguíneo de cavalos não exercitados.

\section{MATERIAL E MÉTODOS}

Oito equinos sem raça definida ( 5 fêmeas e 3 machos castrados), clinicamente sadios, com idade entre seis e 14 anos $(10 \pm 1,68)$, provenientes da mesma região, com altitude inferior a 400 metros, alimentados previamente e, durante o período de estudo, apenas com feno de alfafa foram usados neste experimento. Esses cavalos foram submetidos a 43 sessões de hipóxia normobárica com duração de uma hora durante o mês de Janeiro. A hipóxia foi gerada por um compressor e um módulo computadorizado ${ }^{\mathrm{a}}$ que regula a retirada do oxigênio do ar atmosférico na concentração desejada. Os animais recebiam esse ar com menor $\left[\mathrm{O}_{2}\right]$ através de uma máscara ${ }^{\mathrm{b}}$, que era colocada 15 minutos antes do início de cada sessão. Nos quinze dias que antecederam o experimento, os equinos foram habituados ao ambiente e à máscara, permanecendo com ela uma hora por dia.

Nas três primeiras sessões, foi utilizada uma $\left[\mathrm{O}_{2}\right]$ de $17 \%$, que foi sendo reduzida em $1 \%$ a cada duas sessões até atingir a $\left[\mathrm{O}_{2}\right]$ de $13 \%$, mantida por quatro sessões. A partir desse momento, a $\left[\mathrm{O}_{2}\right]$ foi reduzida a $12 \%$ e mantida por 30 sessões, completando assim as 43 sessões de hipóxia. Com exceção do primeiro dia, no qual os animais foram submetidos a apenas uma sessão, nos dias subsequentes, o regime foi de duas sessões diárias, totalizando 22 dias de tratamento.

Com auxílio de anestesia local com lidocaína a $2 \%$, foi fixado assepticamente um cateter ${ }^{\mathrm{c}} 14 \mathrm{G}$ em uma das veias jugulares de cada cavalo, através do qual foram coletadas as amostras sanguíneas.

Antes do início da primeira sessão de hipóxia e ao final da última, os cavalos foram submetidos ao teste de tolerância à glicose intravenosa (TTGIV). Para isso, foi administrado $0,5 \mathrm{~g}$ de glicose por $\mathrm{kg}$ de peso vivo de uma solução a $50 \%$ durante um período de 10 minutos (MEHRING \& TYZNIK, 1970; DE LACORTE et al., 1999). Amostras de sangue foram coletadas antes e 2, 4, 8, 16, 30, 60, 90, 120, 150 e 180 minutos após a administração de glicose em tubos de ensaio contendo EDTA e fluoreto de potássio para determinação da concentração da glicose plasmática, e em tubos sem anticoagulante para dosagem da insulina sérica. As amostras foram imediatamente centrifugadas após a coleta e armazenadas a $-80^{\circ} \mathrm{C}$ até o momento da determinação dos níveis de glicose e insulina, através 
do teste enzimático de glicose-oxidase e quimioluminescência, respectivamente.

Para determinação sérica de cortisol, foram coletadas amostras de sangue em tubos de ensaio sem anticoagulante durante as sessões 1, 4, 6, 8, 10, 14, 22 e 43. As coletas foram feitas imediatamente antes de colocar a máscara (-15), antes de iniciar a hipóxia (0), e $15,30,45$ e 60 minutos após seu início. Essas amostras foram centrifugadas e o soro armazenado a $-80^{\circ} \mathrm{C}$ até $\mathrm{o}$ momento da análise, através do método de quimioluminescência. Imediatamente antes da coleta de cada amostra, a frequência cardíaca (FC) do cavalo foi determinada por auscultação com estetoscópio.

$\mathrm{O}$ efeito dos distintos fatores nas variáveis dependentes foi analisado utilizando o PROC MIXED (mixed models, SAS Institute Inc., Cary, NC) em um modelo para dados repetidos. Foram determinados os efeitos principais de tratamento e tempo assim como sua interação. $\mathrm{O}$ efeito dos tratamentos sobre as variáveis dependentes em um tempo específico foi determinado pelo uso de ESTIMATES, também do SAS. Os resultados estão representados como média \pm erro padrão da média e o nível de significância adotado foi $\mathrm{P}<0,05$.

\section{RESULTADOS E DISCUSSÃO}

A curva glicêmica dos equinos submetidos ao teste intravenoso de tolerância à glicose não foi alterada pela exposição às 43 sessões de hipóxia (Figura 1A). Por outro lado, a curva de insulina mostrou alteração significativa nos tempos de coleta 2, 4, 8, 16,
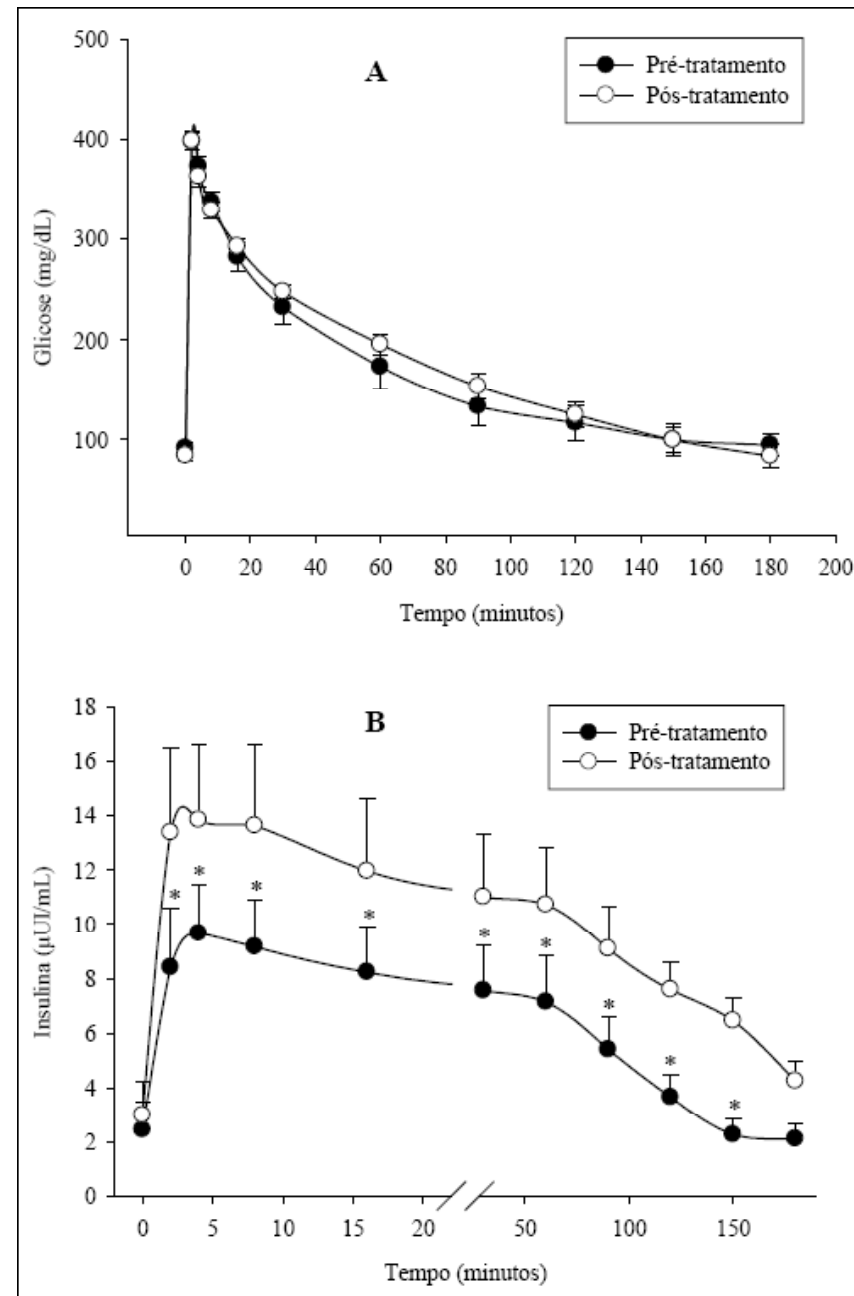

Figura 1 - Resposta glicêmica (A) e secreção de insulina (B) durante o teste de tolerância à glicose intravenosa, realizado antes (Pré-tratamento) e após (Pós-tratamento) as 43 sessões de hipóxia. Os dados são apresentados como média \pm erro padrão da média. * indica diferença significativa entre os mesmos pontos das duas curvas $(\mathrm{P}<0,05)$. 
30, 60, 90, 120 e 150 minutos (Figura 1B) $(\mathrm{P}<0,05)$. Tal achado demonstra que, com exceção das amostras coletadas antes da administração de glicose e daquelas coletadas 180 minutos após, momento em que a glicemia volta aos níveis basais, foram necessários maiores níveis de insulina para manter os mesmos níveis de glicose.

Quando ocorre diminuição da sensibilidade à insulina, há um aumento compensatório de sua secreção pelas células ß pancreáticas (KAHN, 2003; KRONFELD et al., 2005). Foi o que demonstraram BARNHOLT et al. (2006) no homem, após deslocamento do nível do mar para uma altitude de 4.300 metros. Com a exposição à hipóxia de forma contínua, RAFF et al. (1999) obtiveram resultados semelhantes expondo ratos recém-nascidos a uma $\left[\mathrm{O}_{2}\right]$ de $12 \%$ por sete dias. Um aumento da resistência à insulina também foi observado em terneiros submetidos a uma $\left[\mathrm{O}_{2}\right]$ de 4,8 a $5,9 \%$ (CHENG et al., 1997).

Utilizando HI, POLOTSKY et al. (2003) chegaram à mesma conclusão quando submeteram ratos a $5 \%$ de $\left[\mathrm{O}_{2}\right]$, por períodos de 30 segundos seguidos de reoxigenação nos 30 segundos subsequentes, durante 16 horas diárias por até 12 semanas. Entretanto, IIYORI et al. (2007) demonstraram que, com metodologia semelhante, um aumento da resistência à insulina em ratos é obtido com apenas 9 horas de exposição à HI. Não foi encontrada na literatura demonstração de que tal efeito possa ser obtido com o método utilizado no presente estudo.

Embora os mecanismos dessa relação ainda não estejam bem elucidados, um dos fatores ao qual se atribui a diminuição de sensibilidade à insulina pela $\mathrm{HI}$ é a ativação do sistema nervoso simpático (HARSCH, 2004; PLIQUETT et al., 2004) pela liberação de catecolaminas, que podem diminuir a sensibilidade à insulina, diminuir a glicólise e aumentar a gliconeogênese (DEIBERT \& DEFRONZO, 1980). Entretanto, IIYORI et al. (2007) demonstraram que o bloqueio da atividade do sistema nervoso autônomo não atenua tais efeitos, sugerindo que outros mecanismos possam estar envolvidos na alteração do metabolismo pela hipóxia.

Outro fator a considerar é atividade corticotrófica da HI, que pode levar ao aumento do cortisol circulante, decorrente da modificação do eixo HHA (COSTE et al., 2005). Esse hormônio tem a capacidade de aumentar a resistência à insulina, inibir sua secreção pelas células $\beta$ do pâncreas e aumentar a gliconeogênese hepática (LAMBILLOTTE et al., 1997; ANDREWS \& WALKER 1999).
TAYLOR (1998), estudando os efeitos endócrinos e metabólicos de pôneis anestesiados, sendo ou não submetidos a um período de hipóxia induzida, sugeriu que o leve aumento na concentração plasmática de glicose e na frequência cardíaca, observada no grupo que recebeu menos oxigênio, foi consequência do aumento da atividade simpática como uma resposta ao estresse. Entretanto, o mesmo autor observou que os dois grupos apresentaram elevação semelhante nos níveis de cortisol durante a anestesia, sugerindo que a anestesia geral por si só representa uma situação de estresse para a espécie e estimula a resposta máxima do córtex adrenal, visto que não há aumento do hormônio pela ação de hipóxia, cirurgia ou hipercapnia.

Já foi relatado que a hipóxia aguda em humanos em repouso pode aumentar (HUMPELER et al., 1980), ou mesmo não alterar (SUTTON et al., 1977), os níveis de cortisol circulante. Quando há exposição crônica a altitudes elevadas, ocorre um aumento passageiro do cortisol sanguíneo, com retorno aos níveis encontrados ao nível do mar após 30 dias de aclimatização (SAWHNEY et al., 1991).

A concentração de cortisol foi superior na sessão com $\left[\mathrm{O}_{2}\right]$ de $17 \%$ (S1) após 30 minutos de exposição, quando comparado aos valores da sessão com $\left[\mathrm{O}_{2}\right]$ de $13 \%$ (S10) (Figura 2A) $(\mathrm{P}<0,05)$. Segundo HAUGER et al. (1990), no estresse crônico, a exposição repetida ao agente estressor produz uma habituação, ou seja, pode ocorrer uma diminuição na resposta do eixo HHA. Apesar disso, quando um indivíduo recebe estímulos repetidos semelhantes, seguidos de um novo estímulo estressante, a resposta do eixo HHA passa a ser a mesma ou maior do que a resposta inicial (HAUGER et al., 1990; BHATNAGAR \& DALLMAN, 1998). Isso explica o fato de não ter havido diferença nos níveis de cortisol sérico quando a $\left[\mathrm{O}_{2}\right]$ foi reduzida a 12\% (S14) em relação aos da S1 (17\%) (Figura 2A). O estímulo estressante continuou sendo a hipóxia, todavia a $\left[\mathrm{O}_{2}\right]$ pode ter sido baixa o suficiente para representar um novo estímulo estressante. Talvez seja necessário expor esses indivíduos a mais sessões a $13 \%$ de $\left[\mathrm{O}_{2}\right]$ para que o cortisol se mantenha mais baixo ou continue a diminuir.

Houve uma diminuição significativa nos níveis de cortisol a partir dos 15 minutos $(\mathrm{P}<0,05)$ na nona sessão em que os animais foram submetidos a $12 \%$ de $\left[\mathrm{O}_{2}\right](\mathrm{S} 22)$, comparado a $\mathrm{S} 1$ (17\%). Além disso, quando $\mathrm{S} 1$ é comparada com a última sessão do experimento (S43), essa diferença é observada nos tempos -15, 0, 45 e 60 minutos ( $\mathrm{P}<0,05)$ (Figura 2A). Esses dados mostram que a exposição contínua a $12 \%$ de $\left[\mathrm{O}_{2}\right]$ foi capaz de diminuir os níveis de cortisol em 




relação ao início do tratamento. Não foram observadas diferenças entre as coletas feitas na mesma sessão.

Se os achados relativos ao cortisol são indicativos de um evento de estresse, eles são corroborados pela FC dos animais deste estudo. Analisando-se os dados de FC, ocorre um padrão semelhante, com diferença significativa $(\mathrm{P}<0,05) \mathrm{em}$ todos os tempos quando as curvas da S1 são comparadas com a S10. Essa diferença se desfaz quase que totalmente na $\mathrm{S} 14$, momento em que a hipóxia é intensificada a $12 \%$ de $\left[\mathrm{O}_{2}\right]$ e volta a aparecer em alguns pontos na S43 (Figura 2B).

\section{CONCLUSÃO}

A curva glicêmica de equinos clinicamente sadios não submetidos a exercício não é alterada pela exposição a 43 sessões de hipóxia normobárica.
A diminuição da frequência cardíaca e dos níveis de cortisol sugere uma adaptação à exposição crônica da hipóxia que, por sua vez, resultou num aumento de secreção da insulina.

\section{FONTES DE AQUISIÇÃO}

a - PORTABLE-2008 GO2Altitude ${ }^{\circledR}$ Hypoxicator: BIOMEDTECH AUSTRALIA Pty. Ltd. 17 Roberna Street Moorabbin, 3189, Victoria, Melbourne, Australia. b - ERA ${ }^{\oplus}$ Mask: BIOMEDTECH AUSTRÁLIA Pty. Ltda. 17 Roberna Street, Moorabbin, 189, Victoria, Melbourne, Australia. c - A-Cath ${ }^{\oplus}$ : BECTON DICKINSON IND. CIRÚRGICAS LTDA. Av. Pres. Juscelino Kubitschek, 273, Juiz de Fora, Minas Gerais, Brasil.

\section{COMITÊ DE ÉTICA E BIOSSEGURANÇA}

O presente estudo foi aprovado pelo Comitê de Ética e Bem-Estar Animal da Instituição de origem, parecer n.015/2009, e está de acordo com os Princípios Éticos na Experimentação Animal (COBEA). 


\section{REFERÊNCIAS}

AGUILERA, G. Regulation of pituitary ACTH secretion during chronic stress. Frontiers in Neuroendocrinology, v.15, p.321-350, 1994. Disponível em: <http://www.ncbi.nlm.nih.gov/ pubmed/7895891>. Acesso em: 21 dez. 2010.

ALEXANDER, S.; IRVINE, C.H.G. Stress in the racing horse: coping vs not coping. Journal of Equine Science, v.9, n.3, p.77-81, 1998. Disponível em: <http://www.jstage.jst.go.jp/article/ jes/9/3/77/_pdf>. Acesso em: 21 dez. 2010.

ANDREWS, R.C.; WALKER, B.R. Glucocorticoids and insulin resistance: old hormones, new targets. Clinical Science, v.96, p.513-523, 1999. Disponível em: <http://www.clinsci.org/ cs/096/0513/0960513.pdf>. Acesso em: 21 dez. 2010.

BARNHOLT, K.E. et al. Endocrine responses to acute and chronic high-altitude exposure $(4,300$ meters): modulating effects of caloric restriction. American Journal of Physiology Endocrinology and Metabolism, v.290, p.1078-1088, 2006. Disponível em: <http://ajpendo.physiology.org/content/290/6/ E1078.full.pdf+html>. Acesso em: 21 dez. 2010. doi:10.1152/ ajpendo.00449.2005.

BHATNAGAR, S.; DALLMAN, M. Neuroanatomical basis for facilitation of hypothalamic-pituitary-adrenal responses to a novel stressor after chronic stress. Neuroscience, v.84, n.4, p.10251039, 1998. Disponível em: <http://www.ncbi.nlm.nih.gov/ pubmed/9578393>. Acesso em: 21 dez. 2010.

BRAUN, B. et al. Women at altitude: short-term exposure to hypoxia and/or $\alpha 1$-adrenergic blockade reduces insulin sensitivity. Journal of Applied Physiology, v.91, p.623631, 2001. Disponível em: <http://jap.physiology.org/content/ 91/2/623.full.pdf+html>. Acesso em: 21 dez. 2010.

BURTSCHER, M. et al. Intermittent hypoxia increases exercise tolerance in patients at risk for or with mild COPD. Respiratory Physiology \& Neurobiology, v.165, p.97-103, 2009. Disponível em: <http://www.ncbi.nlm.nih.gov/pubmed/19013544>. Acesso em: 21 dez. 2010. doi:10.1016/j.resp.2008.10.012.

CHEN, X. et al. Effects of hypoxia on glucose, insulin, glucagon, and modulation by corticotropin-releasing factor receptor type 1 in the rat. Endocrinology, v.148, n.7, p.3271-3278, 2007. Disponível em: <http://5041.973 program.org/admin/DOC/ thesis/20071204032431.pdf>. Acesso em: 21 dez. 2010. doi: 10.1210/en.2006-1224.

CHENG, N. et al. Effect of hypoxia on blood glucose, hormones, and insulin receptor functions in newborn calves. Pediatric Research, v.41, n.6, p.852-856, 1997. Disponível em: <http:/ /journals.1ww.com/pedresearch/Fulltext/1997/06000/ Effect_of_Hypoxia_on_Blood_Glucose,_Hormones,_and.9.aspx >. Acesso em: $21 \mathrm{dez} .2010$.

COSTE, O. et al. Hypoxic alterations of cortisol circadian rhythm in man after simulation of a long duration flight. Steroids, v.70, p.803-810, 2005. Disponível em: <http:// www.ncbi.nlm.nih.gov/pubmed/16019044>. Acesso em: 21 dez. 2010. doi:10.1016/j.steroids.2005.05.003.

DEIBERT, D.C; DEFRONZO, R.A. Epinephrine-induced insulin resistance in man. Journal of Clinical Investigation, v.65, p.717-721, 1980. Disponível em: <http://www.ncbi.nlm.nih.gov/ pmc/articles/PMC371414/>. Acesso em: 21 dez. 2010. doi: 10.1172/JCI109718.

DE LA CORTE, F.D. et al. Glucose uptake in horses with polysaccharide storage myopathy. American Journal of Veterinary Research, v.60, n.4, p.458-462, 1999. Disponível em: 〈http://www.ncbi.nlm.nih.gov/pubmed/10211689>. Acesso em: 21 dez. 2010.

HARSCH, I.A. Continuous positive airway pressure treatment rapidly improves insulin sensitivity in patients with obstructive sleep apnea syndrome. American Journal of Respiratory Critical Care Medicine, v.169, p.156-162, 2004. Disponível em: <http://ajrccm.atsjournals.org/cgi/content/short/169/2/ 156>. Acesso em: 21 dez. 2010. doi: 10.1164/rccm.200302206OC.

HAUGER, R.L. et al. CRF receptor regulation and sensitization of ACTH responses to acute ether stress during chronic intermittent immobilization stress. Brain Research, v.532, p.34-40, 1990. Disponível em: <http://www.ncbi.nlm.nih.gov/ pubmed/2178035>. Acesso em: $21 \mathrm{dez} .2010$.

HINES, M.T. Clinical approach to commonly encountered problems. In: REED, S.M. et al. Equine internal medicine. 2.ed. Saint Louis: Saunders, 2004. Cap.3, p.111-168.

HUMPELER, E. et al. Influence of exposure to moderate altitude on the plasma concentration of cortisol, aldosterone, rennin, testosterone and gonadotropins. European Journal of Applied Physiology, v.17, p.167-176, 1980. Disponível em: <http:// www.springerlink.com/content/m201845k00842417/>. Acesso em: 21 dez. 2010. doi: 10.1007/BF00421324.

IIYORI, $\mathrm{N}$. et al. Intermittent hypoxia causes insulin resistance in lean mice independent of autonomic activity. American Journal of Respiratory Critical Care Medicine, v.175, p.851-857, 2007. Disponível em: http://ajrccm.atsjournals.org/ cgi/content/full/175/8/851. Acesso em: 21 dez. 2010. doi: 10.1164/rccm.200610-15270C.

KAHN, S.E. The relative contributions of insulin resistance and beta-cell dysfunction to the pathophysiology of Type 2 diabetes. Diabetologia, v.46, p.3-19, 2003. Disponível em: <http://www.springerlink.com/content/lpcd37uvu 7e3v0lb/>. Acesso em: 21 dez. 2010. doi: 10.1007/s00125-002-1009-0.

KRONFELD, D.S. et al. Insulin resistance in the horse: definition, detection, and dietetics. Journal of Animal Science, v.83, p.22-31, 2005. Disponível em: <http://www.biorumen.net/Xfiles/ Cavalos\%203.pdf>. Acesso em: 21 dez. 2010.

LAMBILLOTTE, C. et al. Direct glucocorticoid inhibition of insulin secretion. Journal of Clinical Investigation, v.99, n.3, p.414-423, 1997. Disponível em: <http://www.ncbi.nlm.nih.gov/ pmc/articles/PMC507814/pdf/990414.pdf〉. Acesso em: $21 \mathrm{dez}$. 2010 .

MEERSON, F.Z. et al. Prevention and elimination of heart arrhythmias by adaptation to intermittent high altitude hypoxia. Clinical Cardiology, v 10, p.783-789, 1987. Disponível em: <http://onlinelibrary.wiley.com/doi/10.1002/clc.4960101202/ pdf>. Acesso em: 21 dez. 2010. doi: 10.1002/clc.4960101202. 
MEHRING, J.S.; TYZNIK, W.J. Equine glucose tolerance. Journal of Animal Science, v.30, p.764-766, 1970. Disponível em: <http://jas.fass.org/cgi/reprint/30/5/764>. Acesso em: 21 dez. 2010.

MÖSTL, E.; PALME, R. Hormones as indicators of stress. Domestic Animal Endocrinology, v.23, p.67-74, 2002. Disponível em: 〈http://www.ncbi.nlm.nih.gov/pubmed/12142227〉. Acesso em: $21 \mathrm{dez} .2010$.

NEUBAUER, J.A. Physiological and genomic consequences of intermittent hypoxia invited review: physiological and pathophysiological responses to intermittent hypoxia. Journal of Applied Physiology, v.90, p.1593-1599, 2001. Disponível em: <http://jap.physiology.org/content/90/4/1593.full.pdf+html>. Acesso em: 21 dez. 2010.

PLIQUETT, R.U. et al. Neurohumoral stimulation in type-2diabetes as an emerging disease concept. Cardiovascular Diabetology, v.3, n.4, p.1-8, 2004. Disponível em: <http:// www.ncbi.nlm.nih.gov/pmc/articles/PMC406517/>. Acesso em: 21 dez. 2010. doi: 10.1186/1475-2840-3-4.

POLOTSKY, V.Y. et al. Intermittent hypoxia increases insulin resistance in genetically obese mice. Journal of Physiology, v.552, p.253-264, 2003. Disponível em: <http://jp.physoc.org/ content/552/1/253.full.pdf $>$. Acesso em: 21 dez. 2010. doi: 10.1113/jphysiol.2003.048173.

RAFF, H. et al. The effect of hypoxia on plasma leptin and insulin in newborn and juvenile rats. Endocrine, v.11, n.1, p.37-39, 1999. Disponível em: <http://www.springerlink.com/ content/155k554170760119/>. Acesso em: $21 \mathrm{dez}$. 2010. doi: 10.1385/ENDO:11:1:37.

SAWHNEY, R.C.et al. Glucoregulatory hormones in man at high altitude. European Journal Applied Physiology, v.62, p.286-291, 1991. Disponível em: <http://www.springerlink.com/ content/t4w1823t72721178/>. Acesso em: $21 \mathrm{dez}$. 2010. doi: $10.1007 / \mathrm{BF} 00571554$.

SEREBROVSKAYA, T.V. Intermittent hypoxia research in the former Soviet Union and the Commonwealth of Independent States: history and review of the concept and selected applications. High Altitude Medicine \& Biology, v.3, n.2, p.205-221, 2002. Disponível em: <http:// www.pharmapacific.com/images/Hypoxia_in_USSR.pdf $>$. Acesso em: 21 dez. 2010.

SUTTON, J.R. Effect of acute hypoxia on the hormonal response to exercise. Journal of Applied Physiology, v.42, p587-592, 1977. Disponível em: <http://jap.physiology.org/ content/42/4/587.full.pdf+html>. Acesso em: 21 dez. 2010.

TAYLOR, P.M. Effects of hypoxia on endocrine and metabolic responses to anaesthesia in ponies. Research in Veterinary Science, v.66, p.39-44, 1998. Disponível em: <http:// www.ncbi.nlm.nih.gov/pubmed/10088710>. Acesso em: 21 dez. 2010 .

ZONG, P. et al. Intermittent hypoxic training protects canine myocardium from infarction. Experimental Biology and Medicine, v.229, p.806-812, 2004. Disponível em: <http:// www.ncbi.nlm.nih.gov/pubmed/15337835>. Acesso em: 21 dez. 2010. 